\title{
Reaction $e^{+} e^{-} \rightarrow \omega \pi$ and $\rho(1450)$ and $\rho(1700)$ mesons in quark model
}

\author{
K. Kittimanapun:* K. Khosonthongkee, C. Kobdaj, P. Suebka, and Y. Yan \\ School of Physics, Institute of Science, \\ Suranaree University of Technology, \\ Nakhon Ratchasima, Thailand
}

(Dated: April 26, 2022)

\begin{abstract}
The investigation in the work of the reaction $e^{+} e^{-} \rightarrow \omega \pi^{0}$ in the ${ }^{3} P_{0}$ nonrelativistic quark model reveals that the $e^{+} e^{-} \rightarrow \omega \pi^{0}$ process at the energy region from the $\omega \pi$ threshold to $2.0 \mathrm{GeV}$ is dominated by the two-step process in which the primary $\bar{q} q$ pair first forms $\rho$ and $\rho^{\prime}$ mesons and then the vector mesons decay into $\omega$ and $\pi$. With $\rho(1450)$ and $\rho(1700)$ mainly in $2 S$ and $1 D$ states respectively, the experimental data for the cross section of the reaction $e^{+} e^{-} \rightarrow \omega \pi^{0}$ are well produced in the ${ }^{3} P_{0}$ quark model. The work supports the argument that $\rho(1450)$ is mainly a $2 S$ meson and $\rho(1700)$ a $1 D$ meson.

PACS numbers: 12.39.Jh, 13.66.Bc, 14.40.Cs
\end{abstract}

\section{INTRODUCTION}

Decay modes $\rho \pi$ and $\omega \pi$ are among the most important for the processes of $e^{+} e^{-}$annihilation into hadrons at low energies, giving mainly the $3 \pi$ and $4 \pi$ final states, respectively [1, 2]. These reactions might be used to study the dynamics of light vector mesons, for example, $\rho^{\prime}$ and $\omega^{\prime}$, which may be formed as the intermediate states and decay then into $\omega \pi$ and $\rho \pi$. The information of mesons except the lightest ones is still rather rare because of the lack of high-quality experimental data and also effective theoretical models. In addition, that $\rho^{\prime}$ may decay into $\omega\left(\rho^{\prime} \rightarrow \omega \pi\right)$ and $\omega^{\prime}$ to $\rho\left(\omega^{\prime} \rightarrow \rho \pi\right)[3]$ adds more uncertainties to the understanding of the properties of the intermediate states. The analysis in the work [4] confirms that the uncertainties between the $\omega$-like resonance and $\rho$-like resonance result in calculations with low accuracy.

Recently, experiments have been set up to study the processes of $e^{+} e^{-}$annihilation to $\pi^{0} \pi^{0} \gamma$ at low energies (below $2 \mathrm{GeV}$ ). The SND experiments in the energy region $0.6-0.97 \mathrm{GeV}$ give information of $\rho$ and $\omega$ intermediate state mesons [5, 6]. The reaction of $\omega \pi \rightarrow \pi^{0} \pi^{0} \gamma$ measured in the center mass energies $0.92-1.38 \mathrm{GeV}$ at CMD-2 shows the interference of $\rho(770)$ meson and $\rho(1450)$ meson, which decays into $\omega \pi^{0}$ [7]. However, the SND experiment with the energy up to $1.4 \mathrm{GeV}$ from the threshold [8] revealed that the experimental cross section can be satisfactorily understood with two excited states $\rho^{\prime}(1400)$ and $\rho^{\prime \prime}(1600)$ in which the contribution of the $\rho^{\prime \prime}(1600)$ meason dominates. However, this result contradicts with the theoretical expectation, where $\rho^{\prime}$ and $\rho^{\prime \prime}$ are

*Electronic address: oou_kritsada@hotmail.com 
considered as $2 S$ and $1 D q \bar{q}$ states respectively and the $\rho^{\prime}$ gives a larger contribution.

The intermediate vector mesons in $e^{+} e^{-}$annihilation reactions at low energies could be simple $\bar{q} q$ states, mixtures of $\rho$-like and $\omega$-like mesons, or even hybrid states $(\bar{q} q$ plus one or more gluons). The idea of exotic meson (vector hybrid) 9] has been proposed, but the theoretical results are not in line with the the experimental data. On the other hand, $q \bar{q}$ structured mesons with different radial and orbital excitations have been extensively studied. An earlier work in quark model [10] predicted a series of excited vector mesons, with $\rho(1450)$ and $\omega(1460)$ being the lowest $\rho$-type state with the $2{ }^{3} S_{1}$ excitation which has a large probability to decay into $\omega \pi^{0}$ and the $\omega$-type state with the $1^{3} D_{1}$ excitation, respectively. The predictions are consistent with some experimental data but in strong contrast with the observations of CMD-2 [1] and CLEO [11] which support the $a_{1}(1260)$ dominance in the reaction $e^{+} e^{-} \rightarrow \omega \pi$.

The prediction in the work [10] that the meson $\rho(1450)$ has a bigger probability to decay into $\omega \pi$ than the $\rho$-type mesons with higher masses is not consistent with the results of the SND experiment [8] that the $\rho(1600)$ meson dominates over the $\rho(1400)$ in the reaction $e^{+} e^{-} \rightarrow \omega \pi$. However, the results of the recent work [12] do not contradict the assignment of the $\rho(1450)$ and $\omega(1420)$ to the state $2{ }^{3} S_{1}$. We study in the present work the reaction $e^{+} e^{-} \rightarrow \omega \pi$ at low energies in the nonrelativistic ${ }^{3} P_{0}$ quark model, aiming at a better understanding of the reaction and the properties of the vector mesons $\rho(1450)$ and $\rho(1700)$. The work is arranged as follows: In Section II we study the reaction $e^{+} e^{-} \rightarrow \omega \pi$ in the ${ }^{3} P_{0}$ quark model and compare our results with experimental data. Discussion and conclusions are given in Section III. The transition amplitudes for the reaction of $\rho^{\prime}$ to two S-wave mesons are given in Appendix A while the model parameters are determined in Appendix B and C.

\section{II. $e^{+} e^{-} \rightarrow \omega \pi$ IN ${ }^{3} P_{0}$ QUARK MODEL}

The reactions $e^{+} e^{-} \rightarrow \omega \pi$ may stem from two possible processes, namely, the one-step process where the $e^{+} e^{-}$ pair annihilates into a virtual time-like photon, then the virtual photon decays into a $\bar{q} q$ pair, and finally the $\bar{q} q$ pair is dressed directly by an additional quark-antiquark pair pumped out of the vacuum to form the $\pi \omega$ final state, and the two-step process where the $e^{+} e^{-}$pair annihilates into a virtual time-like photon, then the virtual photon decays into a $\bar{q} q$ pair, and the $\bar{q} q$ pair first form a vector meson and finally the vector meson decay into the $\omega \pi$ final state.

At high energies the reaction $e^{+} e^{-} \rightarrow \omega \pi$ is likely dominated by the one-step process while in the low-energy region, especially close to the threshold, the reactions are expected to be dominated by the two-step process. It is found that the reactions $e^{+} e^{-} \rightarrow \pi \pi, \bar{N} N$ at low energies are dominated by the two-step process [13, 14]. In this work we study the reaction $e^{+} e^{-} \rightarrow \omega \pi$ at energies close to the threshold, assuming that the reaction is dominated by the two-step process.

The transition amplitude of the reactions $e^{+} e^{-} \rightarrow \omega \pi$ in the two step process shown in Fig. 1 takes the form

$$
T=\left\langle\omega \pi\left|V_{\bar{q} q}\right| \rho^{\prime}\right\rangle\left\langle\rho^{\prime}|G| \rho^{\prime}\right\rangle\left\langle\rho^{\prime} \mid \bar{q} q\right\rangle\left\langle\bar{q} q|T| e^{+} e^{-}\right\rangle
$$

where $\left\langle\rho^{\prime} \mid \bar{q} q\right\rangle$ is simply the wave function of the intermediate meson $\rho^{\prime}$, which takes the Gaussian form in the 
work (see Appendix B). $\left\langle\rho^{\prime}|G| \rho^{\prime}\right\rangle$, the Green function describes the propagation of the intermediate meson, and $\left\langle\pi \omega\left|V_{\bar{q} q}\right| \rho^{\prime}\right\rangle$ is the transition amplitude of the intermediate meson $\rho^{\prime}$ decaying to the $\omega \pi$ pair in the ${ }^{3} P_{0}$ nonrelativistic quark model. Considering the energy region in question we include three intermediate mesons $\rho(770), \rho(1450)$ and $\rho(1700)[3]$ in our calculation, and hence have the transition amplitude take explicitly the form

$$
\begin{aligned}
T & =\left\langle\omega \pi\left|V_{\bar{q} q}\right| \rho(770)\right\rangle\langle\rho(770)|G| \rho(770)\rangle\langle\rho(770) \mid \bar{q} q\rangle\left\langle\bar{q} q|T| e^{+} e^{-}\right\rangle \\
& +\left\langle\omega \pi\left|V_{\bar{q} q}\right| \rho(1450)\right\rangle\langle\rho(1450)|G| \rho(1450)\rangle\langle\rho(1450) \mid \bar{q} q\rangle\left\langle\bar{q} q|T| e^{+} e^{-}\right\rangle \\
& +\left\langle\omega \pi\left|V_{\bar{q} q}\right| \rho(1700)\right\rangle\langle\rho(1700)|G| \rho(1700)\rangle\langle\rho(1700) \mid \bar{q} q\rangle\left\langle\bar{q} q|T| e^{+} e^{-}\right\rangle
\end{aligned}
$$

The transition amplitudes are evaluated, following the conventions of the work [14], for the process of the intermediate meson $\rho^{\prime}$ decaying to two mesons. The explicit results are given in Appendix A. The transition amplitude for the process $e^{+} e^{-} \rightarrow \rho^{\prime}$ in eq. (2) can be easily evaluated in the standard method of quantum field theory, taking the form in eq. (B5). The Green function in Eq. (2) describing the propagation of the intermediate meson takes the form

$$
\left\langle\rho^{\prime}|G| \rho^{\prime}\right\rangle=\frac{1}{E_{c m}-\left(M_{\rho^{\prime}}-i \Gamma_{\rho^{\prime}} / 2\right)}
$$

where $M_{\rho^{\prime}}$ and $\Gamma_{\rho^{\prime}}$ are the mass and width of the intermediate meson $\rho^{\prime}$, and $E_{c m}$ is the center-of-mass energy of the system. The total cross section is finally obtained from

$$
\sigma=\frac{2 \pi E_{1} E_{2} k}{4 E_{c m}^{2} p} \int\left|T_{e^{+} e^{-} \rightarrow m_{1} m_{2}}\right|^{2} d \Omega
$$

where $E_{i}, E_{c m}, p, k$ are respectively the energy of the $i$ th final meson, the center-of-mass energy of the system, the incoming and outing momenta in the center of mass system. We intend to study the reaction $e^{+} e^{-} \rightarrow \omega \pi^{0}$

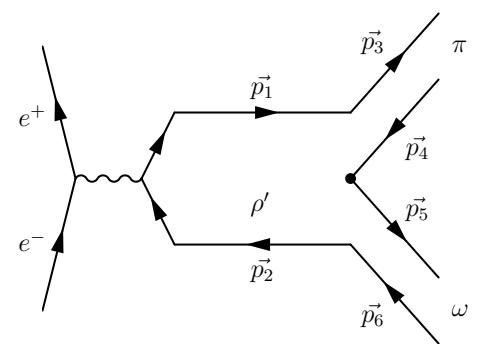

FIG. 1: Reaction $e^{+} e^{-} \rightarrow \omega \pi$ in the two-step process.

in the ${ }^{3} P_{0}$ nonperturbative quark model with all the model parameters predetermined. There are two parameters for the ${ }^{3} P_{0}$ quark model, the meson size parameter and the effective coupling constant of the ${ }^{3} P_{0}$ vertex. In this work we take the meson size parameter $a=3.24 \mathrm{GeV}^{-1}$ as determined by the process $\rho(\omega, \phi) \rightarrow e^{-} e^{+}$in Appendix $\mathrm{B}$, and the ${ }^{3} P_{0}$ effective coupling constant $\lambda=1.25$ as determined by the reaction $\rho \rightarrow \pi \pi$ in Appendix C. In the theoretical calculation we have three intermediate mesons $\rho(770), \rho(1450)$ and $\rho(1700)$ included, with their masses 
and widths taken from the particle data group [3]. While the $\rho(770)$ is kept always as a $1 S$ meson, the $\rho(1450)$ and $\rho(1700)$ are allowed to be the mixtures of the $2 S$ and $1 D$ states, that is

$$
\begin{array}{r}
\rho(1450)=\cos \theta|2 S\rangle+\sin \theta|1 D\rangle \\
\rho(1700)=-\sin \theta|2 S\rangle+\cos \theta|1 D\rangle
\end{array}
$$

where $\theta$ is the mixing angle between the $2 S$ and $1 D$ states.

Shown in Fig. 2 are the theoretical predictions and experimental data for the cross section of the reaction

$e^{+} e^{-} \rightarrow \omega \pi^{0}$. Note that for comparing with the experimental data of the reaction $e^{+} e^{-} \rightarrow \omega \pi^{0} \rightarrow \pi^{0} \pi^{0} \gamma$, we have multiplied our theoretical predictions by the factor 0.087 which is the decay branch ratio of $\omega(780) \rightarrow \pi^{0} \gamma$. In the theoretical study the only free parameter is the mixing angle, with the meson length parameter fixed to be 3.24 by the reaction $\rho \rightarrow e^{+} e^{-}$and the effective coupling constant of the ${ }^{3} P_{0}$ quark vertex fixed to be 1.25 by the reaction $\rho \rightarrow \pi \pi$. In Fig. 2 the dotted curve represents the result with $\rho(770)$ in the $1 S$ state, $\rho(1450)$ in the $1 D$ state and $\rho(1700)$ not included while the dash-dotted curve is for the result with $\rho(770)$ in the $1 S$ state, $\rho(1450)$ in the $1 D$ state and $\rho(1700)$ in the $2 S$ state. It is clear that the $\rho(1450)$ can not be dominated by the $1 D$ component and the $\rho(1700)$ is not allowed by the experimental data to have a large $2 S$ component.

The dashed curve is the theoretical result with $\rho(770)$ in the $1 S$ state and $\rho(1450)$ and $\rho(1700)$ being respectively pure $2 S$ and $1 D$ mesons. The result is fairly consistent with experimental data. Let $\rho(1450)$ and $\rho(1700)$ the mixtures of the $2 S$ and $1 D$ states, one may improve the theoretical results to a certain extent. Shown in Fig. 2 as the solid curve is the theoretical result with $\rho(770)$ in the $1 S$ state, $\rho(1450)$ and $\rho(1700)$ being the states in eq. (5) with $\theta=\pi / 6$. Considering the uncertainty of the experimental data, however, it is difficult to figure out how much D-wave component the $\rho(1450)$ may have.

\section{DISCUSSION AND CONCLUSIONS}

The reaction $e^{+} e^{-} \rightarrow \omega \pi^{0}$ in the energy region from the $\omega \pi$ threshold to $2.0 \mathrm{GeV}$ is investigated in the ${ }^{3} P_{0}$ nonrelativistic quark model with model parameters predetermined by other processes. The experimental data for the cross section of the reaction $e^{+} e^{-} \rightarrow \omega \pi^{0}$ are well reproduced with $\rho(770), \rho(1450)$ and $\rho(1700)$ as the intermediate states, where $\rho(770)$ is in the $1 S$ state, and $\rho(1450)$ and $\rho(1700)$ are dominantly in the $2 S$ and $1 D$ states, respectively.

That the theoretical prediction for the cross section of the reaction $e^{+} e^{-} \rightarrow \omega \pi^{0}$ in the two-step process with the $\rho(770), \rho(1450)$ and $\rho(1700)$ as the intermediate states are consistent with the experimental data at energies below $2.0 \mathrm{GeV}$ leaves no room for the one-step process to contribute to the reaction at a sizable scale at this energy region. The study suggests that at this energy region the two-step process is dominant over the one-step one.

The work strongly suggest that the $\rho(1450)$ is mainly a $2 S$ meson and the $\rho(1700)$ a $1 D$ one. 


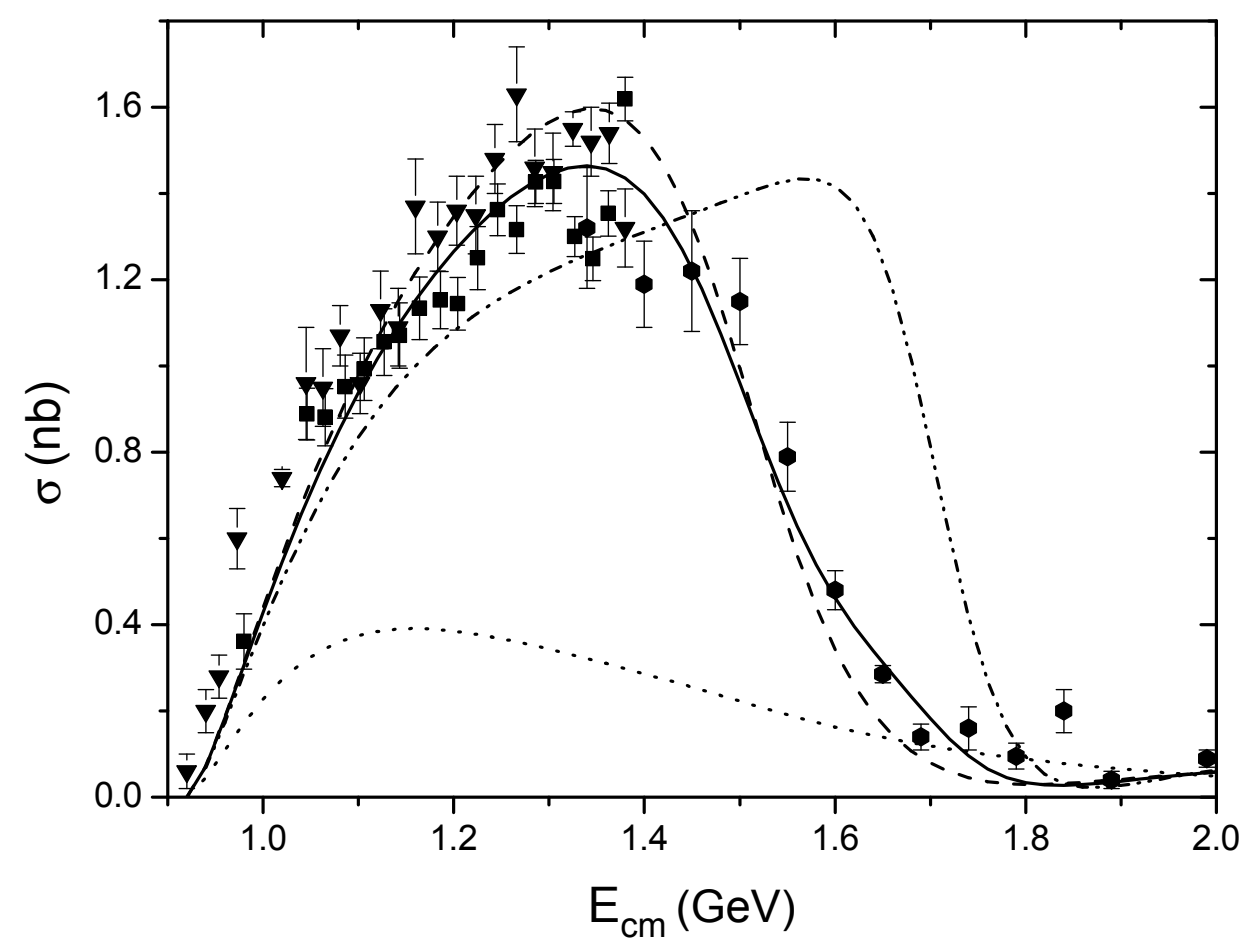

FIG. 2: Theoretical results for the cross section of the reaction $e^{+} e^{-} \rightarrow \omega \pi^{0} \rightarrow \pi^{0} \pi^{0} \gamma$ : the dotted curve for $\rho(1450)$ in $2 S$ state and $\rho(1700)$ not included, the dash-dotted curve for $\rho(1450)$ in $1 D$ state and $\rho(1700)$ in $2 S$ state, the dashed curve for $\rho(1450)$ in the $2 S$ state and $\rho(1700)$ in $1 D$ state, and the solid curve for the case where $\theta=\pi / 6$ in eq. (5). The experimental data are from the CMD2 [1], SND [8] and DM2 [15]

\section{Acknowledgements}

The work is supported by the Commission on Higher Education, Thailand (CHE-RES-RG Theoretical Physics) and the corresponding author is supported in part by the Development and Promotion of Science and Technology Talents Project (DPST), Thailand.

\section{APPENDIX A: TRANSITION OF $\rho^{\prime} \rightarrow \omega \pi$ IN ${ }^{3} P_{0}$ MODEL}

As the essential building blocks for the transition amplitude of the reaction $e^{+} e^{-} \rightarrow \rho\left(\rho^{\prime}\right) \rightarrow \pi \omega$, the transition amplitudes for the processes $\rho^{\prime} \rightarrow \omega \pi$ are derived in the ${ }^{3} P_{0}$ quark model which are described in details in Appendix C. For the purpose of good documentation, we list obviously the amplitudes as follows:

$$
T_{\rho^{\prime} \rightarrow \omega \pi}=f(k) Y_{1, j_{z}-m_{\omega}}(\hat{k})
$$


with

$$
f(k)=A k\left(1-\frac{2 a^{2}}{15} k^{2}\right) e^{-\frac{1}{12} a^{2} k^{2}}
$$

where

$$
A=\lambda \frac{40 \sqrt{2} a^{3 / 2}}{243 \pi^{1 / 4}} \mathrm{C}\left(111 ; j_{z}, m_{\omega}-j_{z}, m_{\omega}\right)
$$

for a $2 S \rho^{\prime}$ decay to $\omega$ and $\pi$ mesons, and

$$
A=\lambda \frac{16 \sqrt{10} a^{3 / 2}}{243 \pi^{1 / 4}} \mathrm{C}\left(111 ; m_{\rho^{\prime}}, m_{\omega}-m_{\rho^{\prime}}, m_{\omega}\right) \mathrm{C}\left(121 ; m_{\rho^{\prime}}, j_{z}-m_{\rho^{\prime}}, j_{z}\right) \mathrm{C}\left(121 ; m_{\omega}-j_{z}, j_{z}-m_{\rho^{\prime}}, m_{\omega}-m_{\rho^{\prime}}\right)
$$

for a $1 D \rho^{\prime}$ decay to $\omega$ and $\pi$ mesons. Here in the above equations $m_{\rho^{\prime}}$ and $m_{\omega}$ are respectively the spin magnetic quantum numbers of the intermediate $\rho^{\prime}$ meson and the final $\omega$ meson, and $j_{z}$ the total magnetic quantum number of the system. The $\mathrm{C}-\mathrm{G}$ coefficients above are in the form $C\left(l_{1} l_{2} l_{3} ; m_{1} m_{2} m_{3}\right)$. In the evaluation the meson spatial wave functions have been taken as the Gaussian form in eq.(B2). The transition amplitudes above differ by a global factor from the ones in the work [16] since we have defined different color matrices for the decay processes in question.

\section{APPENDIX B: MESON SIZE PARAMETER}

Our intention is to study the reaction $e^{+} e^{-} \rightarrow \omega \pi^{0}$ in the ${ }^{3} P_{0}$ nonperturbative quark model with all the model parameters predetermined. In this study there are two model parameters, the meson size parameter and the effective coupling constant of the ${ }^{3} P_{0}$ vertex. The meson size parameter comes with the meson spatial wave functions, which take the Gaussian form as usual,

$$
R_{n l}(p)=\left[\frac{2 a^{3} n !}{\Gamma\left(n+l+\frac{3}{2}\right)}\right](a p)^{l} e^{-\frac{1}{2} a^{2} r^{2}} L_{n}^{l+1 / 2}\left(a^{2} p^{2}\right) .
$$

where $L_{n}^{l+1 / 2}\left(\alpha^{2} r^{2}\right)$ are the associated Laguerre polynomials, $p$ is the magnitude of the relative momentum between the quark and antiquark, and $n, l$ and $a$ are respectively the principle quantum number, orbital quantum number and the mentioned size parameter. For the lowest states $1 S, 2 S$ and $1 D$, we have the meson spatial wave functions

$$
\begin{gathered}
\psi_{s p}=\frac{\sqrt{a^{3}}}{\pi^{3 / 4}} e^{-\frac{1}{2} a^{2} p^{2}}, \\
\psi_{s p}=\frac{1}{\pi^{3 / 4}} \sqrt{\frac{2 a^{3}}{3}}\left(\frac{3}{2}-a^{2} p^{2}\right) e^{-\frac{1}{2} a^{2} p^{2}},
\end{gathered}
$$

and

$$
\psi_{s p}=\frac{4}{\pi^{1 / 4}} \sqrt{\frac{a^{3}}{15}}(a p)^{2} e^{-\frac{1}{2} a^{2} p^{2}} Y_{2 m}(\theta, \phi)
$$


The size parameter may be determined by the reaction $\rho(\omega, \phi) \rightarrow e^{+} e^{-}$. The transition amplitude of the reaction is

$$
\begin{aligned}
T_{\rho \rightarrow e^{+} e^{-}}= & \sum_{\alpha} \sum_{s_{q} s_{\bar{q}}} \sum_{t_{q} t_{\bar{q}}} \frac{1}{\sqrt{3}} C\left(\frac{1}{2} \frac{1}{2} S, s_{q} s_{\bar{q}} S_{z}\right) C\left(\frac{1}{2} \frac{1}{2} I, t_{q} t_{\bar{q}} I_{z}\right) \\
& \cdot \int \frac{d \vec{p}}{(2 \pi)^{3 / 2} 2 E_{q}} \psi_{\rho}(\vec{p}) T_{q \bar{q} \rightarrow e^{+} e^{-}}
\end{aligned}
$$

where $\alpha$ is color indices of the quark and antiquark of the $\rho$ meson, $s_{q}\left(t_{q}\right)$ and $s_{\bar{q}}\left(t_{\bar{q}}\right)$ are the z-axis spin (isospin) projections for quark and antiquark, respectively. $S(I)$ (actually equal to 1 ) and $S_{z}\left(I_{z}\right)$ are respectively the spin (isospin) and the z-axis spin (isospin) projection of the $\rho$ meson. $\psi_{\rho}(\vec{p})$ is the spatial wave function of the $\rho$ meson in momentum space with $\vec{p}$ being the relative momentum between the quark and antiquark inside, and $T_{q \bar{q} \rightarrow e^{+} e^{-}} \equiv\left\langle e^{+} e^{-}|T| q \bar{q}\right\rangle$ is the transition amplitude of the reaction of a quark-antiquark pair to an electron-positron pair, taking the form

$$
\left\langle e^{+} e^{-}|T| q \bar{q}\right\rangle=-\frac{e_{q} e}{s} \bar{u}_{e}\left(p_{e^{-}}, m_{e^{-}}\right) \gamma^{\mu} v_{e}\left(p_{e^{+}}, m_{e^{+}}\right) \bar{v}_{q}\left(p_{\bar{q}}, m_{\bar{q}}\right) \gamma_{\mu} u_{q}\left(p_{q}, m_{q}\right)
$$

where $s=\left(p_{q}+p_{\bar{q}}\right)^{2}, e_{q}$ is the quark charge, and the Dirac spinors are normalized according to $\bar{u} u=\bar{v} v=2 m_{q}$.

We derive the size parameter $a=3.32,3.49$ and $2.92 \mathrm{GeV}^{-1}$ from the reactions $\rho \rightarrow e^{+} e^{-}, \omega \rightarrow e^{+} e^{-}$and $\phi \rightarrow e^{+} e^{-}$, respectively. Here we have used as inputs $\alpha=1 / 137, M_{\rho}=0.775 \mathrm{GeV}, M_{\omega}=0.782 \mathrm{GeV}, M_{\phi}=1.019$ $\mathrm{GeV}$, the quark masses $m_{u}=m_{d}=5 \mathrm{MeV}$ and $m_{s}=100 \mathrm{MeV}$, and the experimental values of $\Gamma_{\rho^{0} \rightarrow e^{+} e^{-}}=7.02$ $\mathrm{keV}, \Gamma_{\omega \rightarrow e^{+} e^{-}}=0.60 \mathrm{keV}$ and $\Gamma_{\phi \rightarrow e^{+} e^{-}}=1.27 \mathrm{keV}$. The averaged value of the meson size parameter is $a=3.24$ $\mathrm{GeV}^{-1}$.

The transition amplitude of the decay process $\rho \rightarrow e^{+} e^{-}$in eq. (B5) is made up of two pieces: the transition amplitude $T_{q \bar{q} \rightarrow e^{+} e^{-}}$of the process $q \bar{q} \rightarrow e^{+} e^{-}$as shown in eq. (B6), and the projection of the quark-antiquark pair to the $\rho$ meson state, which is performed by the summation and integration in eq. (B5). The masses of quark and antiquark appear only in the transition amplitude $T_{q \bar{q} \rightarrow e^{+} e^{-}}$and the spatial wave function $\psi_{\rho}(\vec{p})$ of the $\rho$ meson in momentum space has no direct dependence of quark masses. Therefore, it may be more reasonable to let quarks take the current masses instead of the constituent masses.

\section{APPENDIX C: STRENGTH OF ${ }^{3} P_{0}$ QUARK MODEL}

In this work we study the reaction $e^{+} e^{-} \rightarrow \omega \pi^{0}$ in the nonperturbative quark model with the ${ }^{3} P_{0}$ quark dynamics

which describes the quark-antiquark annihilation and creation. The ${ }^{3} P_{0}$ model, first introduced in the work [17], has made considerable successes in understanding low-energies hadron physics [18, 19, 20, 21].

The ${ }^{3} P_{0}$ decay model defines the quantum states of a quark-antiquark pair destroyed into or created from vacuum to be $J=0, L=1, S=1$ and $T=0$. The effective vertex in ${ }^{3} P_{0}$ model takes the form

$$
V_{i j}=\lambda \vec{\sigma}_{i j} \cdot\left(\vec{p}_{i}-\vec{p}_{j}\right) \hat{F}_{i j} \hat{C}_{i j} \delta\left(\vec{p}_{i}+\vec{p}_{j}\right)
$$




$$
=\lambda \sum_{\mu} \sqrt{\frac{4 \pi}{3}}(-1)^{\mu} \sigma_{i j}^{\mu} Y_{1 \mu}\left(\vec{p}_{i}-\vec{p}_{j}\right) \hat{F}_{i j} \hat{C}_{i j} \delta\left(\vec{p}_{i}+\vec{p}_{j}\right)
$$

where $\sigma_{i j}^{\mu}, \hat{F}_{i j}, \hat{C}_{i j}$, and $\lambda$ are respectively the spin, flavor and color operators, and the effective coupling constant. The operations of flavor, color, and spin operators onto a $q \bar{q}$ pair are

$$
\begin{aligned}
\left\langle 0,0\left|\hat{F}_{i j}\right| T, T_{z}\right\rangle & =\sqrt{2} \delta_{T, 0} \delta_{T_{z}, 0}, \\
\left\langle 0,0\left|\hat{C}_{i j}\right| q_{\alpha}^{i} \bar{q}_{\beta}^{j}\right\rangle & =\delta_{\alpha \beta}, \\
\left\langle 0,0\left|\sigma_{i j}^{\mu}\right|\left[\bar{\chi}_{i} \otimes \chi_{j}\right]_{J M}\right\rangle & =(-1)^{M} \sqrt{2} \delta_{J, 1} \delta_{M,-\mu}
\end{aligned}
$$

where $\chi_{i}\left(\bar{\chi}_{i}\right)$ is the spin state of quark (antiquark), $\alpha$ and $\beta$ the color indices, and $T$ and $T_{z}$ the total isospin and its projection of the $q \bar{q}$.

In this work we determine the effective coupling constant of the ${ }^{3} P_{0}$ quark vertex with the reaction $\rho(770) \rightarrow$ $\pi^{+} \pi^{-}$. The decay width of the reaction $\rho \rightarrow \pi^{+} \pi^{-}$takes the form

$$
\Gamma_{\rho^{0} \rightarrow \pi^{+} \pi^{-}}=\frac{\pi}{4} M_{\rho}^{2} \sqrt{1-\frac{4 M_{\pi}^{2}}{M_{\rho}^{2}}}\left|T_{\rho^{0} \rightarrow \pi^{+} \pi^{-}}\right|^{2}
$$

In the ${ }^{3} P_{0}$ model, the transition amplitude $T_{\rho^{0} \rightarrow \pi^{+} \pi^{-}}$is derived as in the center-of-mass system [14],

$$
T_{\rho^{0} \rightarrow \pi^{+} \pi^{-}}=-\frac{16 \lambda a^{3 / 2} e^{-\frac{1}{12} a^{2} k^{2}} k}{27 \sqrt{3} \pi^{1 / 4}}
$$

where $a$ is the size parameter of the mesons, which appears in the wave functions of the mesons. $k$ in eq. (C4) is the momentum of the final $\pi$ mesons. The transition amplitude differ by a constant factor from the one in the work [16] since we have used different color matrix. Using as an input $a=3.24 \mathrm{GeV}^{-1}$ and experimental data for $M_{\pi}$, $M_{\rho}$ and $\Gamma_{\rho^{0} \rightarrow \pi^{+} \pi^{-}}$taken from [3] to eq. (C44), one derives $\lambda=1.25$.

[1] R.R. Akhmetshin et al., Phys. Lett. B 466, 392 (1999).

[2] R.R. Akhmetshin et al., Phys. Lett. B 595, 101 (2004).

[3] W.M. Yao et al., Review of particle physics, J. Phys. G 33, (2006).

[4] M.N. Achasov et al., Phys. Lett. B 462, 365 (1999).

[5] M.N. Achasov et al., Phys. Lett. B 537, 201 (2002).

[6] R.R. Akhmetshin et al., Phys. Lett. B 613, 29 (2005).

[7] R.R. Akhmetshin et al., Phys. Lett. B 562, 173 (2003).

[8] M.N. Achasov et al., Phys. Lett. B 486, 29 (2000).

[9] A. Donnachie and Yu.S. Kalashnikova, Phys. Rev. D 60, 114011(1999).

[10] S. Godfrey and N. Isgur, Phys. Rev. D 32, 189 (1985).

[11] K.W. Edward et al., Phys. Rev. D 61, 072003 (2000).

[12] N.N. Achasov and A.A. Kozhevnikov, Phys. Rev. D 57, 4334 (1998). 
[13] P. Suebka, C. Kobdaj and Y. Yan, Int. J. Mod. Phys. 14, 7, 987 (2005).

[14] Y. Yan et al., Phys. Rev. C 71, 025204 (2005).

[15] D. Bisello et al., Nucl. Phys. B (Proc. Suppl.) 21, 111 (1991).

[16] T. Barnes, F.E. Close, P.R. Page, and E.S. Swanson, Phys. Rev. D 55, 4157 (1997).

[17] L. Micu, Nucl. Phys. B 10, 521 (1969).

[18] A. Le Yaouanc et al., Phys. Rev. D 8, 2223 (1973); D 9, 1415 (1974); D 11, 1272 (1975).

[19] M. Maruyama, S. Furui and A. Faessler, Nucl. Phys. A 472, 643 (1987); M. Maruyama, S. Furui, A. Faessler and R. Vinh Mau, Nucl. Phys. A 473, 649 (1987); T. Gutsche, M. Maruyama and A. Faessler, Nucl. Phys. A 503, 737 (1989).

[20] C.B. Dover, T. Gutsche, M. Maruyama and A. Faessler, Prog. Part. Nucl. Phys. 29, 87 (1992).

[21] A. Muhn, T. Gutsche, R. Thierauf, Y. Yan and A. Faessler, Nucl. Phys. A 598, 285 (1996). 